\title{
Aprutumab Ixadotin
}

National Cancer Institute

\section{Source}

National Cancer Institute. Aprutumab Ixadotin. NCI Thesaurus. Code C121212.

An antibody-drug conjug ate (ADC) directed ag ainst the fibroblast growth factor receptor type 2 (FGFR2) and conjug ated to an as of yet unidentified toxin, with potential antineoplastic activity. Upon intravenous administration, aprutumab ixadotin binds to FGFR2. Upon binding, the toxin selectively induces cell death, through an as of yet undisclosed mechanism of action, in FGFR2-expressing tumor cells. FGFR2, a receptor tyrosine kinase upregulated in many tumor cell types, plays an essential role in tumor cell proliferation, differentiation and survival. 